\title{
Doggone affordances: Canine perception of affordances for reaching
}

\author{
Jeffrey B. Wagman ${ }^{1}$ - Matthew D. Langley ${ }^{1}$ • Valeri Farmer-Dougan ${ }^{1,2}$
}

Published online: 6 February 2017

(C) Psychonomic Society, Inc. 2016

\begin{abstract}
Performing any behavior requires perceiving affordances-whether and how that behavior can be performed. Perception of affordances exhibits action scalingchoices about when to transition between two different modes of behavior reflect the fit between action capabilities and environmental properties. The boundary between distances that are perceived to be reachable with an arm-only reach and those that are perceived to be reachable with an arm-plustorso reach occurs at farther distances for long-armed than for short-armed people, but at the same ratio of objectdistance-to-arm length for both groups. To the extent that perception of affordances is supported by detection of invariant stimulation patterns, perception of a given affordance ought to exhibit action scaling regardless of species. We investigated the heights at which dogs chose to transition from reaching with the head only to rearing (i.e., reaching with the head plus torso). This transition occurred at a taller height for tall than for short dogs, but at the same ratio of shoulder-height-totreat-height for both groups. The results demonstrate a similarity in perception of affordances across species and suggest that perception of affordances is supported by detection of lawfully structured stimulation patterns that may be invariant across species.
\end{abstract}

Keywords Animal cognition · Embodied cognition · Visual perception

Jeffrey B. Wagman

jeffreywagman@illinoisstate.edu

1 Department of Psychology, Illinois State University, Normal, IL, USA

2 School of Biological Sciences, Illinois State University, Normal, IL, USA
Performing any behavior requires perceiving whether and how that behavior can be performed. Such possibilities for performing behaviors are known as affordances (J. J. Gibson, 1979/2014). Affordances are action scaled-they are determined by the task-specific fit between action capabilities and environmental properties. Over the last few decades, a large body of research has shown that perception of whether and how to perform a given behavior is also action scaled (for reviews, see Dotov, de Wit, \& Nie, 2012; Fajen, Riley, \& Turvey, 2009). In particular, choices about how and when to transition between two different modes of performing the same behavior are determined by this task-specific fit. For example, the boundary between distances that are perceived to be reachable with an arm-only reach and those that are perceived to be reachable with an arm-plus-torso reach occurs at a farther distance for long-armed than for short-armed people, but at the same ratio of object-distance-to-arm length for both groups (Carello, Grosofsky, Reichel, Solomon, \& Turvey, 1989; Mark, Nemeth, Gardner, \& Dainoff, 1997; see Fisher, 2000; Gabbard, Ammar, \& Rodrigues, 2005; Wagman, 2012). Demonstrating that the transition occurs different distances but at a constant ratio for people who differ in action capabilities is critical for establishing action scaling in perception because it shows that perception reflects a task-specific relationship between action capabilities and environmental properties.

From the ecological approach to perception action (J. J. Gibson, 1979/2014), perception of a given affordance is supported by detection of an invariant stimulation pattern that is informative about the task-specific fit between action capabilities and environmental properties. From this perspective, objects and events lawfully structure patterned energy distributions (e.g., the optic array or the acoustic array) such that this structure is specific to its source and 
is available to be detected at a point of observation that a perceiver might occupy (see Petrusz \& Turvey, 2010; Turvey 2002). This lawful structuring of energy distributions entails that the stimulation pattern that is informative about a particular affordance is invariant across both particular instances of perceiving and the particular anatomical components by which the stimulation pattern is detected (Turvey, 1992, 2002). Such invariance putatively underlies the ability to perceive the same affordance with different configurations of the same perceptual modality and by means of entirely different perceptual modalities (see Rosenblum, 2011; Wagman \& Hajnal, 2014, 2016). For example, blindfolded people listening to sound-producing objects presented at different distances also show actionscaled boundaries between distances that are perceived to be reachable with an arm-only reach and those that are perceived to be reachable with an arm-plus-torso reach (Rosenblum, Wuestefeld, \& Anderson, 1996).

Such lawful structuring of energy distributions not only entails that such informative stimulation patterns are invariant across instances of perceiving and across anatomical components but also that they are invariant across species (so long as the ability to detect such structure is comparable across those species; see Shaffer, Krauchunas, Eddy, McBeath, 2004). Accordingly, to the extent that perception of affordances is supported by detection of invariant stimulation patterns, perception of a given affordance ought to exhibit action scaling regardless of species. Of course, a comprehensive investigation of this hypothesis requires investigating perception of affordances for many different behaviors by many different species (e.g., perception of affordances for lever pressing by rats and by hamsters; Cabrera, Sanabria, Jiménez, \& Covarrubias, 2013). However, given recent research on the perceptual, cognitive, communicative, and social abilities of canines (e.g., Hare \& Woods, 2013; Shaffer et al., 2004) and the important roles played by dogs as service animals, we investigated this hypothesis with respect to perception of affordances for reaching by canines.

We systematically presented food treats to canines at various heights and determined the height at which a given canine chose to transition from a head-only reach to rearing (i.e., a head-plus-torso reach). We expected that heights at which individual canines chose to make this transition would be action scaled. In the context of this experiment, action scaling entails that this transition occur at different treat heights but a constant ratio of treat-height-toshoulder-height for dogs that differ in reaching ability. Consequently, we expected that the boundary between treat heights that are perceived to be reachable with a head-only reach and those that are perceived to be reachable by rearing would occur at taller heights for tall dogs than for short dogs, but at the same ratio of treat-height-to-shoulder- height for both groups (cf. Carello et al., 1989; Rosenblum et al., 1996). ${ }^{1}$

\section{Method}

Subjects Nineteen dogs (Canis lupus familiaris) were recruited from a local animal rescue facility, Illinois State student or faculty owners, and the Illinois State University Service Dog Organization (six pets, five service, eight shelter canines). Two of the dogs were deaf. This sample size was based on previous research on perception of affordances for reaching by humans (cf. Carello et al., 1989; Rosenblum, et al., 1996). Body measurements, breeds, ages, status, and sex of individual dogs are listed in Table 1.

Written informed consent was obtained from the owner of each dog in accordance with Illinois State University's Institutional Animal Care and Use Committee guidelines, and the project was approved by the Illinois State University Institutional Animal Care and Use Committee.

Materials and apparatus A small research room $(1.5 \mathrm{~m} \times$ $2.4 \mathrm{~m}$ ) in the main canine training laboratory was used for all trials. A treat delivery apparatus was mounted on the wall directly across from an entry door. Each side of the apparatus consisted of a pair of parallel vertical copper pipes $(1.27 \mathrm{~cm} \times$ $152.4 \mathrm{~cm}$ ), placed one in front of the other such that there was a 2-cm gap between them, and connected with an elbow connector. The pairs of pipes were placed $50 \mathrm{~cm}$ apart, and each end of the apparatus was secured to the wall with bracket and screws. A horizontal crossbar (another copper pipe, $1.27 \mathrm{~cm} \times$ $60 \mathrm{~cm}$ ) was fitted into the vertical track created by the two sides of the apparatus. A measuring cup (1 cup, GoodCook) was secured to the bottom of the crossbar. The height of the crossbar was controlled by the experimenter by means of a string and pulley system attached to the ceiling. A tape measure secured to the wall allowed the experimenter to measure the height of the measuring cup (see Fig. 1).

Procedure At the beginning of the experiment, a leashed canine was brought into the experimental room by the trainer or owner and prompted to sit or stand in front of the wallmounted apparatus. On the initial familiarization trial of the experiment, a food treat (chopped hot dogs, Great Value, Wal-

\footnotetext{
${ }^{1}$ Although the ecological approach to perception-action proposes that optical information that supports perception of a given affordance is available to be detected at a point of observation (in this case, the eye height of the dog, cf. Mark, 1987), our analyses are with respect to the shoulder height of the dog because, to a large degree, this height constrains the maximum heights that can be reached with the head only and by rearing. Hence, evaluating our hypothesis that the height at which individual canines chose to transition from one means of reaching to another will be action scaled requires coding choices with respect to these anthropometric variables (cf. Carello et al., 1989; Rosenblum et al., 1996).
} 
Table 1 Subject characteristics

\begin{tabular}{|c|c|c|c|c|c|c|}
\hline Breed & Age (mos.) & Sex & Status & Shoulder height $(\mathrm{cm})$ & Hip height $(\mathrm{cm})$ & Chest-to-tail length $(\mathrm{cm})$ \\
\hline Labrador Mix & 7 & Male & Pet & 53.3 & 59.7 & 53.3 \\
\hline Golden Retriever & 8 & Male & Service & 53.3 & 69.9 & 53.3 \\
\hline Standard Poodle & 24 & Female & Service & 62.2 & 59.7 & 63.5 \\
\hline Golden Retriever & 36 & Male & Service & 68.6 & 76.2 & 68.6 \\
\hline Pyrenees Mix & 10 & Male & Service & 64.8 & 63.5 & 63.5 \\
\hline Shepherd Mix & 30 & Male & Pet & 59.7 & 73.7 & 61.0 \\
\hline Terrier Mix & 7 & Male & Shelter & 48.3 & 55.9 & 48.3 \\
\hline Labrador/ Terrier & 8 & Female & Shelter & 53.3 & 58.4 & 53.3 \\
\hline Blue Heeler & 24 & Male & Shelter & 55.9 & 63.5 & 55.9 \\
\hline Shepherd & 54 & Male & Disabled & 59.7 & 73.7 & 61.0 \\
\hline Rottweiler & 48 & Female & Pet & 63.5 & 73.7 & 63.5 \\
\hline Shepherd/ Pyrenees & 60 & Male & Disabled & 55.9 & 55.9 & 55.9 \\
\hline Shepherd & 5 & Male & Service & 52.1 & 59.7 & 63.5 \\
\hline Rottweiler & 12 & Male & Pet & 58.4 & 66.0 & 58.4 \\
\hline Australian Cattle & 15 & Male & Service & 50.8 & 50.8 & 61.0 \\
\hline Terrier & 12 & Female & Service & 49.5 & 50.8 & 49.5 \\
\hline Terrier Mix & 108 & Female & Service & 50.8 & 61.0 & 50.8 \\
\hline Labrador & 24 & Male & Service & 57.2 & 62.2 & 57.2 \\
\hline Beagle & 84 & Male & Service & 41.9 & 48.3 & 41.9 \\
\hline Mean $(S D)$ & 30.3 & & & $55.6(6.4)$ & $62.2(8.3)$ & $62.2(8.3)$ \\
\hline
\end{tabular}

Mart brand), was placed into the cup that was adjusted to be at the canine's eye level. The canine was then prompted to eat the food treat via manual and verbal gestures by the experimenter. Once this occurred, the food treat was replaced. This procedure was repeated until the canine ate the food treat without prompting (typically 3-5 replacements). Once the
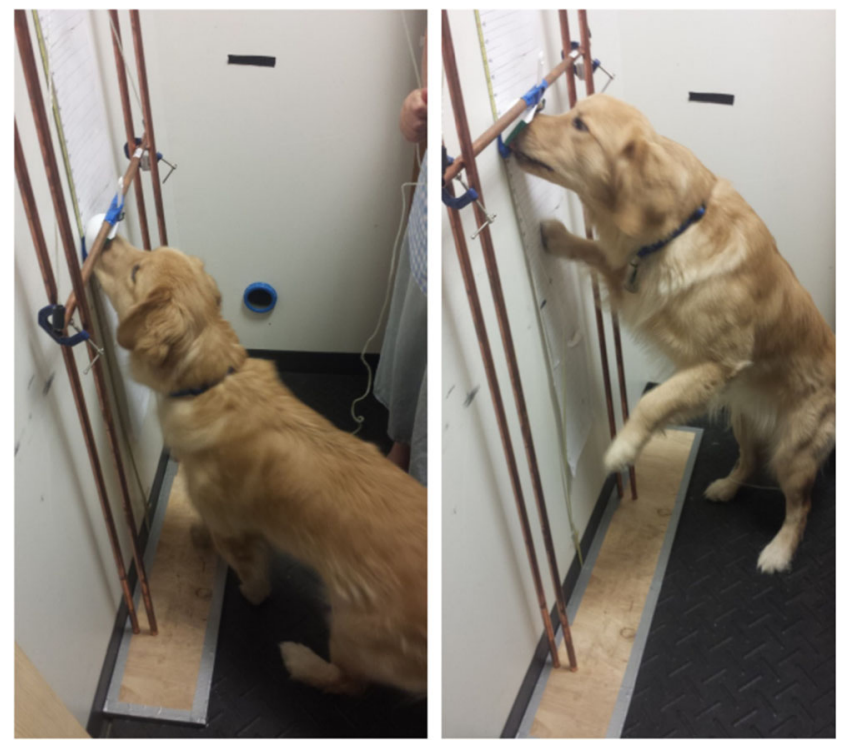

Fig. 1 The experimental apparatus, and examples of reaching with the head only (left) and rearing (right) treat retrieval behavior was established, the canine was prompted to sit or stand approximately 0.5 meters away from the apparatus.

The experimenter then placed another food treat into the cup and raised the cup approximately $5 \mathrm{~cm}$ from its initial height, via the pulley system. The canine was again prompted to eat the food treat. This process was repeated until the canine no longer attempted to reach for the food treat with the head only (see Fig. 1, left) and instead attempted to reach for it by rearing. A rear was defined as the dog raising itself on its hind legs with both front paws completely off of the ground (see Fig. 1, right). Raising only one front paw off of the ground did not change the height of the animal's head (or reaching ability) and therefore was not classified as a rear. The experimenter then lowered the food container approximately $1.25 \mathrm{~cm}$. This process was repeated until the canine no longer attempted to reach for the food treat by rearing and instead reached for it with the head only. The experimenter then raised the food container by a $1.25 \mathrm{~cm}$ to reestablish the rearing behavior.

The minimum height at which rearing occurred as determined by this procedure was the rearing boundary for that animal. At minimum, this procedure required three adjustments of treat height. However, most dogs required between five and seven adjustments to establish the rearing boundary. Rearing boundaries for each dog satisfied the criteria that (1) rearing occurred twice at this particular height and (2) rearing did not occur one increment $(1.25 \mathrm{~cm})$ below this height. Once 
the rearing boundary was determined for a given animal, the experiment ended. The experimenter then measured shoulder height (vertical distance from the ground to the highest point of the scapula), hip height (vertical distance from ground to highest point on pelvis), and chest-to-tail length (from tip of sternum to pelvis) of the dog.

\section{Results}

Dogs were divided into tall and short groups post hoc on the basis of shoulder height. Dogs with shoulder heights less than $58.0 \mathrm{~cm}$ were placed into the short group $(n=12)$ and dogs with shoulder heights equal to or greater than $58.0 \mathrm{~cm}$ were placed into the tall group $(n=7)$. This shoulder height boundary falls between the range of shoulder heights for American Kennel Club categories of medium (approximately $44 \mathrm{~cm}-$ $51 \mathrm{~cm}$ ) and large (approximately $58 \mathrm{~cm}-66 \mathrm{~cm}$ ) size dog breeds. An independent samples $t$ test confirmed that the mean shoulder height of dogs in the short group was shorter $(M=$ $51.9 \mathrm{~cm}, 95 \% \mathrm{CI}=49.5 \mathrm{~cm}-54.2 \mathrm{~cm}$ ) than that of dogs in the tall group $(M=62.4 \mathrm{~cm}, 95 \% \mathrm{CI}=59.8 \mathrm{~cm}-65.0 \mathrm{~cm}), t(17)=$ $5.65, p<.001$, Cohen's $d=2.75$.

Mean rearing boundaries (in $\mathrm{cm}$ ) for the two groups of dogs were compared with an independent samples $t$ test. Rearing boundaries occurred at a taller height for dogs in the tall group $(M=95.2 \mathrm{~cm}, 95 \% \mathrm{CI}=90.8 \mathrm{~cm}-99.6 \mathrm{~cm})$ than for those in the short group $(M=80.3 \mathrm{~cm}, 95 \% \mathrm{CI}=76.6 \mathrm{~cm}-84.0 \mathrm{~cm})$, $t(17)=4.95, p<.001$, Cohen's $d=2.38$ (see Fig. 2A). In addition, a linear regression of rearing boundary on shoulder height (including all dogs from both height groups) showed that there was a strong positive correlation between these variables, with over $90 \%$ of the variance in mean rearing boundary accounted for by shoulder height $\left(r^{2}=.91, p<.001\right.$; see Fig. 2B).

The preceding analyses established that the boundary between food treats that are perceived to be reachable with a head-only reach and those that are perceived to be reachable by rearing occurs at taller heights for tall than for short dogs. Demonstrating action scaling additionally requires establishing that this boundary occurs at the same ratio of treat-heightto-shoulder-height for tall and for short dogs. The subsequent set of analyses investigated whether this was, in fact, the case.

Mean rearing boundaries for each dog were divided by the shoulder height of that dog. Ratio values for the two groups of dogs were compared with an independent samples $t$ test. There was no difference between these ratios for dogs in the tall group $(M=1.53 \mathrm{~cm}, 95 \% \mathrm{CI}=1.50-1.55)$ and those in the short group $(M=1.55 \mathrm{~cm}, 95 \% \mathrm{CI}=1.51-1.59), t(17)=$ $0.910, p>.250$, Cohen's $d=0.46$ (see Fig. 2C). In addition, a linear regression of these ratios on shoulder height (including all dogs in both height groups) showed that there was no significant correlation between these variables, with less than
$10 \%$ the variance in mean rearing boundary accounted for by ratio of rearing-boundary-to-shoulder height $\left(r^{2}=.07, p>\right.$ .250; see Fig. 2D).

\section{General discussion}

A large body of research has shown that perception of affordances is action scaled (see Dotov et al., 2012; Fajen et al., 2009). In particular, choices about how and when to transition between two different modes of performing a given behavior reflect the task-specific fit between action capabilities and environmental properties. However, much of this research has focused on perception of affordances by humans (but see Cabrera et al., 2013; Sonoda, Asakura, Minoura, Elwood, \& Gunji, 2012). Other research has thoroughly investigated the perceptual, cognitive, communicative, and social abilities of canines (e.g., Hare \& Woods, 2013; Shaffer et al., 2004), but little, if any, of this work has explicitly investigated perception of affordances. Such work seems particularly valuable given the important roles played by dogs as service animals. This experiment is a step toward filling this gap in the literature.

The results showed that canine perception of affordances for reaching is action scaled. In particular, the boundary between food treat heights that were perceived to be reachable with a head-only reach and those that were perceived to be reachable by rearing occurred at taller heights for tall dogs than for short dogs, but at the same ratio of treat-height-toshoulder-height for both groups. Such results suggest that perception reflects a task-specific relationship between action capabilities and environmental properties. In large part, such results are analogous to those from investigations of perception of reaching boundaries in humans, which show that the boundary between distances that are perceived to be reachable with an arm-only reach and those that are perceived to be reachable with an arm-plus-torso reach occur at a farther distance for long-armed than for short-armed people, but at the same ratio of object-distance-to-arm length for both groups (see Carello et al., 1989; Rosenblum et al., 1996; but see below). This similarity in perception of affordances across human and canine species is also consistent with the findings that these species use analogous navigation strategies to intercept and catch flying objects, such as baseballs and Frisbees (Shaffer et al., 2004). Overall, the results support the general hypothesis that perception of affordances is supported by detection of invariant stimulation patterns that are informative about the task-specific fit between action capabilities and environmental properties.

Detection of such invariant patterns likely underlies comparable abilities to perceive a given affordance by means of different perceptual modalities or with different configurations of the same perceptual modality. For example, whether a 

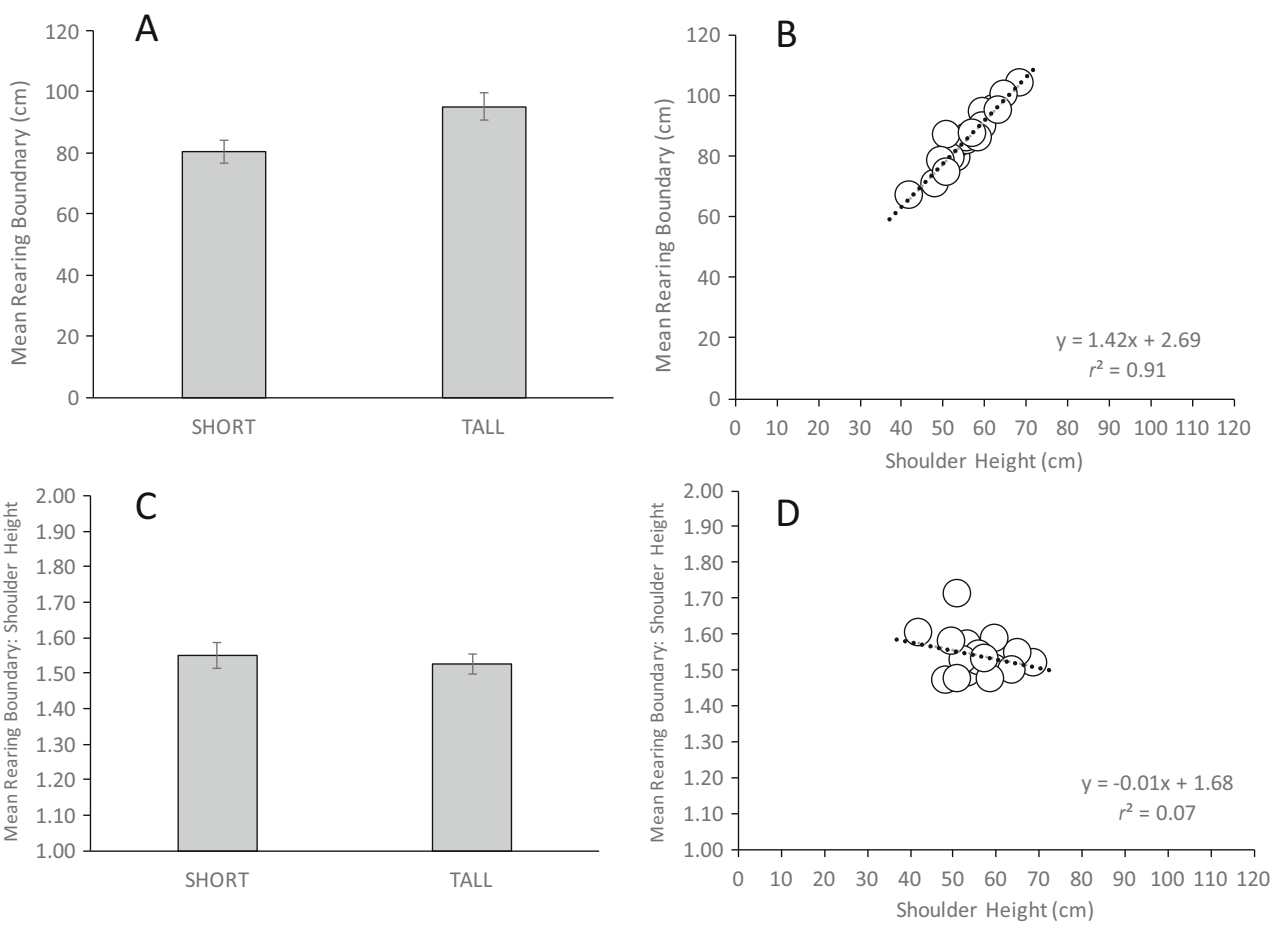

Fig. 2 (A). Mean rearing boundaries (in $\mathrm{cm}$ ) for short and tall dogs. (B) The relationship between mean rearing boundary (in $\mathrm{cm}$ ) and shoulder height for all dogs. (C) Mean ratio of rearing-boundary-to-shoulder-

surface affords standing on can be perceived when a person views that surface or when a blindfolded person probes that surface with a rod held in either hand, attached to either foot, or even attached to the head (Fitzpatrick, Carello, Schmidt, \& Corey, 1994; Wagman \& Hajnal, 2014, 2016). Such results suggest that, with respect to perceiving a given affordance, the particulars of a given energy array may be irrelevant so long as that array can be lawfully structured by the relevant animalenvironment relationship. The results of this experiment build on and extend such findings by suggesting that, with respect to perceiving a given affordance, the particulars of a given nervous system and brain may be irrelevant, so long as the animal can detect the lawful structure in that array that is informative about the relevant animal-environment relationship (see Turvey, 2013). More conservatively, the results of the experiment reported here suggest that the ability to perceive a given affordance may not depend on a particular kind of brain and nervous system (Cabrera et al., 2013; Sonoda et al., 2012; see Shaffer et al., 2004).

\section{Limitations and future directions}

To some extent, rearing can be viewed as the canine equivalent of reaching with the arm-plus-torso. In both cases, transitioning to the respective mode of reaching requires recruiting additional biomechanical degrees of freedom and results in a less stable reaching posture. Of course, rearing and reaching with the arm-plus-torso are not

height for short and tall dogs. (D) The relationship between rearingboundary-to-shoulder-height and shoulder height for all dogs. Error bars represent $95 \%$ confidence intervals.

completely identical. Among the important differences between these behaviors is the source of the resultant postural instability in each case. In humans, the instability is largely a result of the center of mass being shifted away from the base of support (see Fisher, 2000). In canines, however, the instability is largely a result of balancing on the hind legs for the duration of the reach. ${ }^{2}$ As a result, in this experiment, many of the canines placed one or both forepaws against the wall while rearing to retrieve the food treat (see Fig. 1, right). However, for a number of reasons, we do not believe that this behavior influenced the results reported here. First, the analyses focused on transitions from reaching with the head to rearing (and not transitions from rearing to some other behavior, such as jumping). Second, the presentation of treats was initially scaled to the height of the dog and was increased and decreased incrementally from this height. Third, rearing and placing the forepaws against the wall does not serve to increase vertical reaching height above and beyond that of rearing alone. The fact that both canine and human perception of affordances for reaching reflects a task specific fit between action capabilities and environmental properties despite such differences between the reaching behaviors used by these species may

\footnotetext{
${ }^{2}$ Rearing by canines may, perhaps, be more analogous to a human reaching from one foot or while standing on tip toes. Importantly, human perception of affordances for reaching under such circumstances also scales to action capabilities (see Fisher, 2000; Wagman, 2012).
} 
only serve to strengthen the support for the claim that perception of this affordance is supported by detection of a task-specific stimulation patterns that is invariant across species.

As described in the introduction, over the last few decades there has been a great deal of research on perception of affordances by humans and comparatively little on perception of affordances by nonhumans - in particular, on perception of affordances by canines (see Dotov et al., 2012; Fajen et al., 2009; Turvey, 2013). Therefore, there is much ground to be covered in future research. For example, research on perception of affordances by humans has shown that there are developmental changes in perception of affordances across the lifespan (e.g., Konczak, Meeuwsen, \& Cress, 1992; van der Meer, 1997). In particular, such research has shown differences between younger adults and older adults in perception of affordances for reaching (Chateauroux \& Wang, 2008; Ishak, Franchak, \& Adolph, 2014). What qualifies as an older adult dog and a younger adult dog varies with both breed and size (Aldrich, 1995). Given that the sample of dogs in this experiment is quite varied in breed, size, and age, it is not possible to investigate such differences with this sample. However, this is an important topic for future research.

In addition, research on perception of affordances by humans has also shown that maximum reaching distance tends to be overestimated (Carello et al., 1989; Fischer, 2000; Heft, 1993), but tends to increasingly reflect action capabilities with repeated perceptual experience (Ramenzoni, Davis, Riley, \& Shockley, 2008; Wagman, 2012). Given that our methodology required presenting the treat at a height that was scaled to each dog and resulted in one data point per participant (the rearing boundary), it is not possible to investigate either phenomena with the current data. Again, however, this is an important topic for future research.

\section{Conclusion}

Affordances are among the most fundamental relationships between animal and environment and play primary roles in shaping both evolution of species and ontogenetic development of individuals (Reed, 1996; E. J. Gibson \& Pick, 2000). Moreover, in large part, scientific psychology is the science of agency - the ability to select, perceive, and actualize affordances appropriately based on intention (E. J. Gibson, 1994). We propose that investigating perception of affordances over the variety of circumstances in which they are encountered, and by the variety of species that encounter them, will aid in developing a more general scientific psychology and thus more closely align psychology with the natural sciences (Turvey, 2013; Wagman, 2010).

\section{References}

Aldrich, J. (1995). Geriatrics. In M. Siegal (Ed.), UC book of dogs (pp. 58-65). New York, NY: HarperCollins.

Cabrera, F., Sanabria, F., Jiménez, Á. A., \& Covarrubias, P. (2013). An affordance analysis of unconditioned lever pressing in rats and hamsters. Behavioural Processes, 92, 36-46.

Carello, C., Grosofsky, A., Reichel, F. D., Solomon, H. Y., \& Turvey, M. T. (1989). Visually perceiving what is reachable. Ecological Psychology, 1(1), 27-54.

Chateauroux, E., \& Wang, X. (2008). Effects of age, gender, and target location on seated reach capacity and posture. Human Factors: The Journal of the Human Factors and Ergonomics Society, 50(2), 211226.

Dotov, D. G., de Wit, M. M., \& Nie, L. (2012). Understanding affordances: History and contemporary development of Gibson's central concept. AVANT, 3(2), 28-39.

Fajen, B. R., Riley, M. A., \& Turvey, M. T. (2009). Information, affordances, and the control of action in sport. International Journal of Sport Psychology, 40(1), 79.

Fisher, M. H. (2000). Estimating reachability: Whole body engagement or postural stability. Human Movement Science, 19, 297-318.

Fitzpatrick, P., Carello, C., Schmidt, R. C., \& Corey, D. (1994). Haptic and visual perception of an affordance for upright posture. Ecological Psychology, 6(4), 265-287.

Gabbard, C., Ammar, D., \& Rodrigues, L. (2005). Perceived reachability in hemispace. Brain \& Cognition, 58, 172-177.

Gibson, E. J. (1994). Has psychology a future? Psychological Science, $5(2), 69-76$.

Gibson, J. J. (2014). The ecological approach to visual perception: Classic edition. New York, NY: Psychology Press. (original work published in 1979).

Gibson, E. J., \& Pick, A. D. (2000). An ecological approach to perceptual learning and development. New York, NY: Oxford University Press.

Hare, B., \& Woods, V. (2013). The genius of dogs: How dogs are smarter than you think. New York, NY: Penguin.

Heft, H. (1993). A methodological note on overestimates of reaching distance: Distinguishing between perceptual and analytical judgments. Ecological Psychology, 5, 255-271.

Ishak, S., Franchak, J. M., \& Adolph, K. E. (2014). Perception-action development from infants to adults: Perceiving affordances for reaching through openings. Journal of Experimental Child Psychology, 117, 92-105.

Konczak, J., Meeuwsen, H. J., \& Cress, M. E. (1992). Changing affordances in stair climbing: The perception of maximum climbability in young and older adults. Journal of Experimental Psychology: Human Perception and Performance, 18, 691.

Mark, L. S. (1987). Eyeheight-scaled information about affordances: a study of sitting and stair climbing. Journal of Experimental Psychology: Human Perception and Performance, 13, 361-370.

Mark, L. S., Nemeth, K., Gardner, D., \& Dainoff, M. J. (1997). Postural dynamics and the preferred boundary for visually guided reaching. Journal of Experimental Psychology: Human Perception \& Performance, 23, 1365-1379.

Petrusz, S. C., \& Turvey, M. T. (2010). On the distinctive features of ecological laws. Ecological Psychology, 22(1), 44-68.

Ramenzoni, V., Davis, T., Riley, M. A., \& Shockley, K. (2008). Perceiving action boundaries: Learning effects in perceiving maximum jumping-reach affordances. Attention, Perception \& Psychophysics, 72, 1110-1119.

Reed, E. S. (1996). Encountering the world: Toward an ecological psychology. New York, NY: Oxford University Press.

Rosenblum, L. D. (2011). See what I'm saying: The extraordinary powers of our five senses. New York, NY: W. W. Norton \& Company. 
Rosenblum, L. D., Wuestefeld, A. P., \& Anderson, K. L. (1996). Auditory reachability: An affordance approach to the perception of sound source distance. Ecological Psychology, 8(1), 1-24.

Shaffer, D. M., Krauchunas, S. M., Eddy, M., \& McBeath, M. K. (2004). How dogs navigate to catch Frisbees. Psychological Science, 15(7), 437-441.

Sonoda, K., Asakura, A., Minoura, M., Elwood, R. W., \& Gunji, Y. P. (2012). Hermit crabs perceive the extent of their virtual bodies. Biology Letters, 8(4), 495-497.

Turvey, M. T. (1992). Affordances and prospective control: An outline of the ontology. Ecological Psychology, 4(3), 173-187.

Turvey, M. T. (2002). Perception: The ecological approach. Encyclopedia of Cognitive Science. doi:10.1002/0470018860.s00525

Turvey, M. T. (2013). Ecological perspective on perception-action: What kind of science does it entail? In W. Prinz, M. Beisert, \& A. Herwig (Eds.), Action science: Foundations of an emerging discipline (pp. 139-170). Cambridge, MA: MIT Press. van der Meer, A. L. (1997). Visual guidance of passing under a barrier. Early Development and Parenting, 6, 149-158.

Wagman, J. B. (2010). What is responsible for the emergence of order and pattern in psychological systems? Journal of Theoretical and Philosophical Psychology, 30(1), 32.

Wagman, J. B. (2012). Perception of maximum reaching height reflects impending changes in reaching ability and improvements transfer to unpracticed reaching tasks. Experimental Brain Research, 219, 461-476.

Wagman, J. B., \& Hajnal, A. (2014). Task specificity and anatomical independence in perception of properties by means of a wielded object. Journal of Experimental Psychology: Human Perception and Performance, 40(6), 2372.

Wagman, J. B., \& Hajnal, A. (2016). Use your head! Perception of action possibilities by means of an object attached to the head. Experimental Brain Research, 234(3), 829-836. 